\title{
Web2Touch 2018
}

\section{Semantic technologies in smart information sharing and web collaboration}

\author{
Rodrigo Bonacin \\ CTI Renato Archer and \\ UNIFACCAMP \\ Campinas, SP Brazil \\ rodrigo.bonacin@cti.go \\ v.br
}

\author{
Mariagrazia Fugini \\ Dipartimento di \\ Elettronica, \\ Informazione e \\ Bioingegneria \\ Politecnico di Milano \\ Milano, Italy \\ mariagrazia.fugini@poli \\ mi.it
}

\author{
Riccardo Martoglia \\ Dipartimento di Scienze \\ Fisiche, Informatiche e \\ Matematiche \\ Università degli Studi di \\ Modena e Reggio \\ Emilia \\ Modena, Italy \\ riccardo.martoglia@uni \\ mo.it
}

\author{
Olga Nabuco \\ CTI Renato Archer \\ Campinas, SP Brazil \\ olga.nabuco@cti.gov.br
}

\begin{abstract}
We present Web2Touch 2018, one of the Tracks at the 27th IEEE WETICE Conference. Web2Touch 2018 includes five full papers and one short paper tackling very upto-date issues in information sharing and collaboration, including, among others, big data analytics, development of virtual agents and assistants, privacy and security analysis and evaluation. Papers come from areas such as knowledge engineering, linked data, big data, security, safety, and web science. The overall focus is on how research on semantics coupled with crucial topics such as big data analysis, privacy, knowledge representation, and enterprise contents management, among the others, can improve services and collaboration and push forward new ways of interpreting the role of the web.
\end{abstract}

Keywords- semantic web; knowledge representation; collaborative web; linked data; ontology, security, safety, data analytics.

\section{INTRODUCTION}

Web2Touch, since its early days, is committed to bring solutions for collaborative work using the Web. New advances in Web-based systems are the driver for changes in cooperative activities, risk facing, actions in cybersystems and in smart environments, where human activities are performed preferably via the internet and the Web. Web practitioners, users and applications exploit, in rapidly varying ways, the richness of the Web to support the user activities. Based on these considerations, the Web2Touch track tackles knowledge engineering based on software and artificial intelligence, and new methods for Knowledge Organization Systems (e.g. ontologies, taxonomies, etc.), with a focus on information creation, maintenance, disambiguation and other techniques on the Web. We also consider reasoning and deep learning techniques, since these lead to better decisions or awareness of events. Web2Touch deepens the concept of "working together" by exploring themes such as: decision support assistance, collective intelligence, semantic search, smart environments and
Internet of Things (IoT), intentions-based analysis, and other collaborative-based ways for problem solving. W2T wants to both explore the state-of-the-art on these topics, and report user experience and advanced research topics about future collaborative approaches..

Web2Touch 2018 collects papers concerning enhanced organization and management of knowledge. It presents models and tools to represent dynamic changes in shared information, smart and context-aware Web applications, and new domains of application of semantic techniques, such as Industry 4.0, social networks, big data, IoT, enhanced connectivity, and mobile technologies. Web2Touch 2018 is also about practical experiences in both stable and emerging interdisciplinary applications, including smart cities, smart companies, entertainment, security and privacy of mobile applications, and digital agriculture.

The papers of this edition aim at presenting alternatives to deal with the following practical issues:

- Developing solutions for document management systems in innovative big data analytics, including the areas of data acquisition, data storage, and data analysis.

- Proposing approaches to unify the linked data representations by merging duplicates, aiming to acquire a single homogenized object by merging the conflicting information of the linked individual objects (i.e., data fusion approaches).

- Analyzing in a comprehensive way risks of privacy leakage due to in-application advertisements on Android applications, considering aspects such as threats/attacks, security weaknesses, assumptions of adversary model, and threats/attacks due to embedded adware.

- Calculating the Return on Security Investment (ROSI) and analyze the impact of a single security investment upon the whole business by considering its effect upon all critical assets, as well as 
considering uncertainty aspects present related to threat occurrence;

- Exploring semantic technologies in the development of context-aware interactive virtual agents, which are able to detect users' emotional state and express affective behavior aiming to improve their empathy;

- Designing cognitive assistants by exploring complex system modeling, semantic modeling, collective intelligence, and ubiquitous technology.

\section{SELECTED PAPERS}

\section{A. Full Papers}

The paper "Innovative Big Data Analytics: A System for Document Management", by Mariagrazia Fugini and Jacopo Finocchi, introduces a Big Data framework for document management. The paper presents requirements and a reference architecture of the project "Sistema Innovativo Big Data Analytics', as well as the logical model, phases and tools and the architecture of an enterprise management system in a network of companies. The paper presents a real industrial case solution for big data and document management.

The paper "Argumentation-based Explanation of Linked Data Fusion" by Fatiha Saïs, Rallou Thomopoulos, and Andreson Carlos Ferreira Da Silva proposes an approach for merging conflicting information of the linked individual objects (data fusion) based on multi-criteria decision and argumentation. The purpose is to help tracking the rationale for data fusion and to investigate how users can achieve a good understanding of data fusion results. In addition, an argumentation-based approach adds confidence and consistency to the information provided to users.

The paper "Ease or Privacy? A Comprehensive Analysis on Android Embedded Adware" by Anum Javaid, Imran Rashid, Haider Abbas and Mariagrazia Fugini deals with privacy problems related to in-application advertisements of Android applications. This paper proposes an attack/threat model based on confidentiality, integrity and availability aspects. Data collected is quantitatively analyzed supporting conclusions about a theoretical framework that correlates embedded adware, advertisement attacks, and preventive measures.

The paper "Framework for Calculating Return on Security Investment (ROSI) for Security Oriented Organizations" by Haider Abbas, Tahreem Yaqoob, and Azka Arshad, proposes a framework for calculating return on security investment. The proposed framework includes six phases: asset identification and analysis, vulnerability and threat identification, likelihood and impact determination, countermeasure analysis, and ROSI calculation. The framework was validated using dataset from Common Vulnerability Security Systems.

The paper "Exploring Ontologies to Improve the Empathy of Interactive Bots", by Andrey Victor Justo, Felipe Rodrigues Jensen, Ivo Calado, Julio Cesar dos Reis, and Rodrigo Bonacin, proposes a sematic Web-based architecture for improving the virtual agents' (Bots) interpretation capacities, as well as their behaviors and actions. This architecture allows the interpretation of several types of data from the environment, including the users' emotional state. Ontologies and SWRL rules are used to infer affective responses, aiming to improve the Bots empathy. The Bots can benefit from the analysis of multiple inputs (e.g., voice, images, videos) to detect the emotional status of the user and act accordingly.

\section{B. Short Papers}

The paper "A Framework for Designing Cognitive Assistants to Support Agricultural Production Systems", by Julio Cesar dos Reis, Rodrigo Bonacin, Andreiwid Correa, Francisco Edeneziano D. Pereira, Ivo Pierozzi Jr., Sergio A. Torquato, Jarbas L. Cardoso Jr., Katia R. E. Jesus, and Olga Fernanda N. Araujo, presents a framework to design cognitive assistant-based solutions. Software assistants are able to act collaboratively with human agents in favor of collective intelligence. This framework is based on complex system modeling techniques, semantic technology, collaborative systems design, and ubiquitous technology.

\section{WeB2TOUCH TEAM}

\section{A. Program Committee}

We could count upon the precious work done by the members of our Program Committee in reviewing all papers contributing to improve Web2Touch content.

Haider ABBAS, National University of Sciences \& Technology (NUST), Pakistan

Frederic ANDRES, National Institute of Informatics, Japan

Ismael BOUASSIDA, ReDCAD, University of Sfax, Tunisia

Paulo Marcos Siqueira BUENO, University of Campinas, Brazil

Sylvie CALABRETTO, LIRIS, University of Lion, France

Wojciech CELLARY, Poznan University of Economics, Poland

Raja CHIKY, ISEP, France

Marcos DA SILVEIRA, LIST, Luxembourg

Giuseppe DE RUVO, University of Auckland, New Zealand

Julio Cesar DOS REIS, Computer Science Institute, Unicamp, Brazil

André Ricardo Abed GREGIO, Federal University of Paraná, Brazil

Sergio ILARRI, University of Zaragoza, Spain

Dilvan MOREIRA, ICMC, USP, Brazil

Cédric PRUSKI, LIST, Luxembourg

Ivan RICARTE, FT-Unicamp, Brazil

Ramon SALVADOR VALLÉS, UPC-Barcelona Tech, Spain

Lina SOUALMIA, Université de Rouen et CHU de Rouen, France 
B. Chairs

- $\quad$ Rodrigo BONACIN, CTI, Brazil

- Mariagrazia FUGINI, Politecnico di Milano, Italy

- Riccardo MARTOGLIA, UniMoRe, Italy

- Olga-NABUCO, CTI, Brazil 